\title{
ON FINITE ABELIAN GROUPS REALIZABLE AS MISLIN GENERA
}

\author{
Peter Hilton and Dirk Scevenels
}

\begin{abstract}
We study the realizability of finite abelian groups as Mislin genera of finitely generated nilpotent groups with finite commutator subgroup. In particular, we give criteria to decide whether a finite abelian group is realizable as the Mislin genus of a direct product of nilpotent groups of a certain specified type. In the case of a positive answer, we also give an effective way of realizing that abelian group as a genus. Further, we obtain some non-realizability results.
\end{abstract}

\section{Introduction}

The (Mislin) genus ([5]) of a finitely generated nilpotent group $N$, denoted $\mathcal{G}(N)$, is the set of isomorphism classes of finitely generated nilpotent groups $M$ having, at each prime $p$, a localization isomorphic with that of $N$, i.e. $M_{p} \cong N_{p}$ for all $p$. It was shown in [2], [5] that $\mathcal{G}(N)$ may be given the structure of a finite abelian group, with the isomorphism class of $N$ as identity element, if the commutator subgroup $[N, N]$ is finite. Thus we are led to study the class $\mathcal{N}_{0}$ of finitely generated nilpotent groups with finite commutator subgroup.

No general method has yet been discovered for calculating $\mathcal{G}(N)$ when $N \in \mathcal{N}_{0}$. However, in [1] , a general method was given if $N \in \mathcal{N}_{1}$, where $\mathcal{N}_{1}$ is the following subclass of $\mathcal{N}_{0}$. Here we describe $\mathcal{N}_{1}$ by introducing the short exact sequence

$$
T N \longmapsto N \rightarrow F N
$$

associated with the nilpotent group $N$, where $T N$ is the torsion subgroup of $N$ and $F N$ is the torsion-free quotient. Plainly the class $\mathcal{N}_{0}$ is given by the conditions that $T N$ be finite and $F N$ free abelian of finite rank. 
Then the class $\mathcal{N}_{1} \subseteq \mathcal{N}_{0}$ is given by the supplementary conditions

(1) $T N$ is abelian;

(2) $T N \longmapsto N \rightarrow F N$ splits on the right, so that $N$ is the semidirect product for an action $\omega: F N \rightarrow$ Aut $T N$;

(3) $\omega(F N)$ lies in the centre of Aut $T N$.

Moreover, in the presence of (1), condition (3) is equivalent to

(3') given $\xi \in F N$, there exists a positive integer $u$ such that the action of $\xi$ on $T N$ is given by $\xi \cdot a=u a$ for all $a \in T N$ (here, $T N$ is written additively).

Let $t$ be the height of $\operatorname{ker} \omega$ in $F N$, that is,

$$
t=\max \{h \in \mathbb{N} \mid \operatorname{ker} \omega \subseteq h F N\}
$$

(here, $F N$ is written additively). Then it is shown in $[\mathbf{1}]$ that

$$
\mathcal{G}(N) \cong(\mathbb{Z} / t)^{*} /\{ \pm 1\}
$$

where $(\mathbb{Z} / t)^{*}$ is the multiplicative group of units of $\mathbb{Z} / t$. It was further shown how to associate with every unit $m$ of $\mathbb{Z} / t$ a group $N_{m}$ in the genus of $N$ such that

$$
N_{m} \leftrightarrow[m]
$$

provides an isomorphism (1.1). Moreover, an algorithm was given for calculating $t$, knowing the exponent of $T N$ and the positive integers $u$ referred to in (3').

Unfortunately, the class $\mathcal{N}_{1}$ is very restricted; indeed, it was shown in [4] that, if a group $N$ in $\mathcal{N}_{1}$ has non-trivial genus, then $F N$ is cyclic. However, in [3], the systematic calculation of $\mathcal{G}(N)$ was extended from $\mathcal{N}_{1}$ to the class $\mathcal{N}_{2}$ consisting of direct products of groups in $\mathcal{N}_{1}$. It is plain that conditions (1) and (2) for membership of $\mathcal{N}_{1}$ are inherited by direct products, but, in general, condition (3) is not. Thus the class $\mathcal{N}_{2}$ is substantially larger than $\mathcal{N}_{1}$. Of course, membership of $\mathcal{N}_{0}$ is inherited by direct products.

The calculation of $\mathcal{G}(N)$, for $N$ in $\mathcal{N}_{2}$, is somewhat technical, but, from our point of view in this paper, the salient facts are the following. First if $N=N_{1} \times \cdots \times N_{k}$ (all $N_{i} \in \mathcal{N}_{1}$ ) and if $F N_{i}$ is not cyclic for some $i$, then $\mathcal{G}(N)$ is trivial; indeed, we will generalize this result below (see Corollary 2.2). Now assume that $F N_{i}$ is cyclic for all $i(1 \leq i \leq k)$, and, in accordance with (1.1), suppose

$$
\mathcal{G}\left(N_{i}\right) \cong\left(\mathbb{Z} / t_{i}\right)^{*} /\{ \pm 1\}
$$


Let $t=\operatorname{gcd}\left(t_{1}, \ldots, t_{k}\right)$ and let $T_{t}=\left\{p_{1}, \ldots, p_{\lambda}\right\}$, where

$$
t=p_{1}^{\ell_{1}} \ldots p_{\lambda}^{\ell_{\lambda}}, \quad \ell_{j} \geq 1
$$

is the prime power factorization of $t$. Then we determine from the 'fine structure' of the groups $N_{1}, \ldots, N_{k}$ a subset $P$ of $T_{t}$-any subset of $T_{t}$ may arise - so that $\mathcal{G}(N)$ is determined as follows:

Theorem 1.1. The genus $\mathcal{G}(N)$ is obtained from $(\mathbb{Z} / t)^{*}$ by factoring out -1 and those residues $m \bmod t$ such that (see (1.2))

$$
m \equiv \begin{cases} \pm 1 \bmod p_{j}^{\ell_{j}} & \text { if } p_{j} \in P \\ 1 \bmod p_{j}^{\ell_{j}} & \text { if } p_{j} \notin P .\end{cases}
$$

Notice that $\mathcal{G}(N)$ is entirely determined by the two invariants $(t, P)$. It is not difficult to show that, given $(t, P)$ with $P \subseteq T_{t}$, there is always a group $N$ in $\mathcal{N}_{2}$ yielding the invariants $(t, P)$ - see Section 4 . We remark that if $k=1$, so that $N \in \mathcal{N}_{1}$, then $P$ is empty. For full details see [3, Theorem 1.6].

Our principal aim in this paper is to describe those finite abelian groups which arise as described in Theorem 1.1 and which can therefore be realized as a (Mislin) genus group $\mathcal{G}(N)$, for some nilpotent group $N$ in $\mathcal{N}_{2}$. We will thereby also obtain some non-realizability results.

In Section 2 we obtain some preliminary results which are of independent interest. We adopt the convention that $\mathbb{Z} / n$ is written $C_{n}$ when thought of as a multiplicative group.

\section{Preliminary results}

As in Section 1, let $N=N_{1} \times N_{2} \times \cdots \times N_{k}$, where each $N_{i} \in \mathcal{N}_{1}$. There is then a function $\Psi_{i}: \mathcal{G}\left(N_{i}\right) \longrightarrow \mathcal{G}(N)$, given by

$$
\Psi_{i}\left(M_{i}\right)=N_{1} \times \cdots \times N_{i-1} \times M_{i} \times N_{i+1} \times \cdots \times N_{k}
$$

Proposition 2.1. The function $\Psi_{i}$ of (2.1) is a surjective homomorphism.

Proof: In accordance with (1.1) we have the short exact sequence

$$
\{ \pm 1\} \longmapsto\left(\mathbb{Z} / t_{i}\right)^{*} \rightarrow \mathcal{G}\left(N_{i}\right)
$$


that can be embedded in the commutative diagram

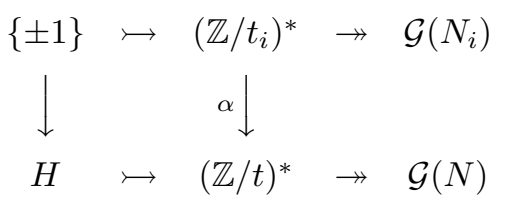

where $H$ is the subgroup factored out of $(\mathbb{Z} / t)^{*}$ to yield $\mathcal{G}(N)$ and $\alpha(m)=m \bmod t$. Then (2.2) may be completed by a homomorphism $\Psi_{i}: \mathcal{G}\left(N_{i}\right) \longrightarrow \mathcal{G}(N)$ which will be surjective since $\alpha$ is surjective. It remains to show that $\Psi_{i}$ is given by (2.1). Of course, we may assume that $F N_{i}$ is cyclic, say, $F N_{i}=\left\langle\xi_{i}\right\rangle$. Then (see [1]) we have a commutative diagram

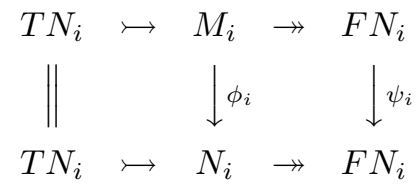

where $\psi_{i}\left(\xi_{i}\right)=m \xi_{i}, m$ being given by $M_{i} \leftrightarrow[m]$ under the isomorphism (1.1) between $\mathcal{G}\left(N_{i}\right)$ and $\left(\mathbb{Z} / t_{i}\right)^{*} /\{ \pm 1\}$. We may, and shall, choose $m$ from its residue class mod $t_{i}$ to be prime to the order of $T N$. Let $T$ be the set of primes $p$ such that $T N$ has $p$-torsion. Set $M=N_{1} \times \cdots \times N_{i-1} \times M_{i} \times N_{i+1} \times \cdots \times N_{k}$. The homomorphisms $\phi_{i}$, $\psi_{i}$ determine, in an obvious way, homomorphisms

$$
\phi: M \longrightarrow N, \quad \psi: F N \longrightarrow F N,
$$

yielding a commutative diagram

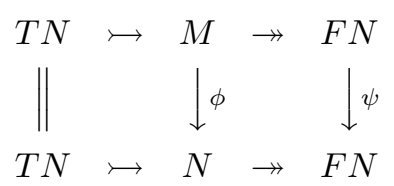

and $\psi$ is a $T$-automorphism with $\operatorname{det} \psi=m$. Thus, in the bottom row of $(2.2),[m] \in(\mathbb{Z} / t)^{*}$ goes to $M$ in $\mathcal{G}(N)$, completing the proof.

Corollary 2.2. Let $N_{1}, \ldots, N_{k} \in \mathcal{N}_{1}$ and set $N=N_{1} \times \cdots \times N_{k}$. Then $\mathcal{G}(N)=0$ if $\mathcal{G}\left(N_{i}\right)=0$ for any $i$.

From the explicit description in [3] of the set of primes $P$ which appears in our statement of Theorem 1.1 the following conclusions are plain. 
Proposition 2.3. Any finite abelian group realizable as $\mathcal{G}(N)$ with $N \in \mathcal{N}_{2}$, is realizable as $\mathcal{G}\left(N_{1} \times N_{2}\right)$, where $N_{1}, N_{2} \in \mathcal{N}_{1}$.

This will simplify our choice of examples in Section 3.

Proposition 2.4. Let $N_{1}, \ldots, N_{k} \in \mathcal{N}_{1}$ and set $N=N_{1} \times \cdots \times N_{k}$. Then $\mathcal{G}\left(N \times N_{j}\right)$ for $1 \leq j \leq k$ is obtained from Theorem 1.1 (applied to $\mathcal{G}(N))$ by taking $P$ to be $T_{t}$ itself. In particular, $\mathcal{G}\left(N \times N_{j}\right)$ is independent of $j$.

\section{Realizing an abelian group as a Mislin genus}

We first enunciate two relevant lemmas on finite abelian groups. For these lemmas we will adopt additive notation; and $p$ will always denote a prime.

Lemma 3.1. Let $G=\bigoplus_{i=1}^{\lambda} \mathbb{Z} / m_{i}$, where $m_{i}=p^{r_{i}+1} n_{i}, r_{i} \geq 0$, $p \nmid n_{i}$, and let $r_{1}=\min _{i} r_{i}$. Let $a_{i}$ be a generator of $\mathbb{Z} / m_{i}$. If $\bar{G}$ is obtained from $G$ by adding the relation $\sum_{i=1}^{\lambda} p^{r_{i}} n_{i} a_{i}=0$, then

$$
\bar{G} \cong \bigoplus_{i=1}^{\lambda} \mathbb{Z} / \bar{m}_{i}=\left\langle\bar{a}_{1}, a_{2}, \ldots, a_{\lambda}\right\rangle,
$$

where

$$
\bar{m}_{i}= \begin{cases}p^{r_{1}} n_{1}, & i=1 \\ m_{i}, & i \geq 2 .\end{cases}
$$

Proof: We have $\mathbb{Z} / m_{1}=\mathbb{Z} / p^{r_{1}+1} \oplus \mathbb{Z} / n_{1}=\left\langle b_{1}, c_{1}\right\rangle$, where $b_{1}=n_{1} a_{1}$, $c_{1}=p^{r_{1}+1} a_{1}$. Then $p^{r_{1}} n_{1} a_{1}=p^{r_{1}} b_{1}$, so the new relation is given by $p^{r_{1}} b_{1}+\sum_{i=2}^{\lambda} p^{r_{i}} n_{i} a_{i}=0$, or

$$
p^{r_{1}}\left(b_{1}+\sum_{i=2}^{\lambda} p^{r_{i}-r_{1}} n_{i} a_{i}\right)=0 .
$$

Set $b^{\prime}=b_{1}+\sum_{i=2}^{\lambda} p^{r_{i}-r_{1}} n_{i} a_{i}$. Then

$$
G=\left\langle b^{\prime}, c_{1}, a_{2}, \ldots, a_{\lambda}\right\rangle=\mathbb{Z} / p^{r_{1}+1} \oplus \mathbb{Z} / n_{1} \oplus \mathbb{Z} / m_{2} \oplus \cdots \oplus \mathbb{Z} / m_{\lambda},
$$

and the new relation is $p^{r_{1}} b^{\prime}=0$. Thus

$$
\bar{G}=\left\langle\overline{b^{\prime}}, c_{1}, a_{2}, \ldots, a_{\lambda}\right\rangle=\mathbb{Z} / p^{r_{1}} \oplus \mathbb{Z} / n_{1} \oplus \mathbb{Z} / m_{2} \oplus \cdots \oplus \mathbb{Z} / m_{\lambda},
$$

and we set $\bar{a}_{1}=\overline{b^{\prime}}+c_{1}$.

Our second lemma is very elementary; the proof will be omitted. 
Lemma 3.2. Let $G=\mathbb{Z} / p \oplus B$, where the first summand is generated by $a$, and let $b \in B$ with $p b=0$. If we obtain $\bar{G}$ from $G$ by adding the relation $a+b=0$, then $\bar{G} \cong B$.

Both these lemmas will be applied with $p=2$. We now apply Theorem 1.1 to prove our main theorem. We denote the Euler totient function by $\Phi$.

Theorem 3.3. The finite abelian groups which are realizable as the genus of a group in $\mathcal{N}_{2}$ are precisely the groups of the form

$$
C_{2^{\ell}} \times \prod_{p_{i} \in P} C_{\frac{1}{2} \Phi\left(p_{i}^{\ell_{i}}\right)} \times \prod_{p_{j} \in Q} C_{\Phi\left(p_{j}^{\ell_{j}}\right)},
$$

where $\ell \geq 0, \ell_{i} \geq 1, \ell_{j} \geq 1$ and $P, Q$ are disjoint (finite) sets of odd primes.

Proof: We will prove that the finite abelian groups which are realizable as the genus of a group in $\mathcal{N}_{2}$ are precisely those groups which, in multiplicative notation, are obtained through the following process:

Step 1: Take $\prod_{i=1}^{\lambda} C_{\Phi\left(p_{i}^{\ell_{i}}\right)}$, where the $p_{i}$ are distinct odd primes and $\ell_{i} \geq 1$.

Step 2: Reduce the order of $\mu$ of the factors $C_{\Phi\left(p_{i}^{\ell_{i}}\right)}$ to $\frac{1}{2} \Phi\left(p_{i}^{\ell_{i}}\right)$, $0 \leq \mu \leq \lambda$.

Step 3: Take the direct product of the result of Step 2 with $C_{2^{\ell}}$, $\ell \geq 0$.

We recall from Theorem 1.1 that $N=N_{1} \times \cdots \times N_{k}$ determines a certain natural number $t$ and that $\mathcal{G}(N)$ is obtained from $(\mathbb{Z} / t)^{*}$ by factoring out the residue class -1 and residue classes $m$ such that $m \equiv \pm 1 \bmod p_{i}^{\ell_{i}}$ for $p_{i} \in P$, where $P$ is a certain subset (perhaps empty) of $T_{t}$, the set of prime divisors of $t=\prod_{i=1}^{\lambda} p_{i}^{\ell_{i}}$, and $m \equiv 1 \bmod p_{i}^{\ell_{i}}$ for $p_{i} \in T_{t}-P$. Obviously, this is equivalent to factoring out -1 and the residue classes $m_{i}$, where $p_{i} \in P$ and

$$
m_{i} \equiv\left\{\begin{array}{l}
-1 \bmod p_{i}^{\ell_{i}} \\
1 \bmod p_{j}^{\ell_{j}}, \quad j \neq i .
\end{array}\right.
$$

Assume first that $t$ is odd, so that each $p_{i}$ is odd. Then $(\mathbb{Z} / t)^{*}$ is given by Step 1. Factoring out $m_{i}$ simply reduces $C_{\Phi\left(p_{i}^{\ell_{i}}\right)}$ to $C_{\frac{1}{2} \Phi\left(p_{i}^{\ell_{i}}\right)}$; it follows from Lemma 3.1 that factoring out -1 reduces $C_{\Phi\left(p_{j}^{\ell_{j}}\right)}$ to $C_{\frac{1}{2} \Phi\left(p_{j}^{\ell_{j}}\right)}$, where 
$p_{j}$ is chosen among the primes in $T_{t}-P$ to be such that the 2-valuation of $p_{j}-1$ is minimal. If $P=T_{t}$, then this last part of Step 2 is void (because then $\left.-1=\prod_{p_{i} \in T_{t}} m_{i}\right)$. Step 3 is also void if $t$ is odd, that is, we take $\ell=0$.

Assume now that $t$ is even. Notice that if $t=2 t^{\prime}$, with $t^{\prime}$ odd, then $(\mathbb{Z} / t)^{*} \cong\left(\mathbb{Z} / t^{\prime}\right)^{*}$ and the process proceeds just as above with $\left(\mathbb{Z} / t^{\prime}\right)^{*}$, using the same subset $P$ and ignoring the prime 2 . Thus we may assume that $4 \mid t$; and we change notation to write

$$
t=2^{\ell+2} \prod_{i=1}^{\lambda} p_{i}^{\ell_{i}}, \quad \ell \geq 0 .
$$

Then

$$
(\mathbb{Z} / t)^{*} \cong C_{2} \times C_{2^{\ell}} \times \prod_{i=1}^{\lambda} C_{\Phi\left(p_{i}^{\ell_{i}}\right)} .
$$

To pass to $\mathcal{G}(N)$, we first factor out the $m_{i}$ defined as in (3.1) with $p_{i} \in P$. This is achieved by a partial Step 2 of the process, applied to $\prod_{i=1}^{\lambda} C_{\Phi\left(p_{i}^{\ell_{i}}\right)}$. If $2 \in P$, we erase $C_{2}$ on the right of (3.2) and then factor out -1 (if $P \neq T_{t}$ ) just as in the case of $t$ odd, by reducing the order of a suitable $C_{\Phi\left(p_{j}{ }_{j}\right)}$ with $p_{j} \in T_{t}-P$. If $2 \notin P$, then we apply Lemma 3.2, factoring out -1 by effectively erasing $C_{2}$. We are thus left with the direct product of $C_{2^{\ell}}, \ell \geq 0$, and the result of Step 2 applied to $\prod_{i=1}^{\lambda} C_{\Phi\left(p_{i}^{\ell_{i}}\right)}$.

We see, conversely, that every group achieved by executing the three steps is realizable as $\mathcal{G}(N)$ with $N \in \mathcal{N}_{2}$-but certainly not uniquely. There is not even always a unique pair $(t, P)$ giving rise to a given finite abelian group. However, if the group we want to realize is

$$
A=C_{2^{\ell}} \times \prod_{p_{i} \in P} C_{\frac{1}{2} \Phi\left(p_{i}^{\ell_{i}}\right)} \times \prod_{p_{j} \in Q} C_{\Phi\left(p_{j}^{\ell_{j}}\right)},
$$

where $P, Q$ are disjoint finite sets of odd primes, then we realize $A$ by the pair $(t, P)$, where

$$
t=2^{\ell+2} \prod_{p_{i} \in P} p_{i}^{\ell_{i}} \prod_{p_{j} \in Q} p_{j}^{\ell_{j}}
$$

and $2 \notin P$ (of course, $P$ or $Q$ may be empty). This completes the proof.

We close this section with two observations supplementary to Theorem 3.3. First we characterize those finite abelian groups which can be realized as $\mathcal{G}(N)$ for $N$ in $\mathcal{N}_{1}$. We recall that this is equivalent to characterizing the finite abelian groups which can be realized as $\mathcal{G}(N)$ for $N \in \mathcal{N}_{2}$ with $P$ empty. This provides the proof of the following. 
Proposition 3.4. The finite abelian groups which are realizable as the genus of a group in $\mathcal{N}_{1}$ are precisely those groups which, in multiplicative notation, are obtained through the following process:

Step 1: Take a group $\prod_{i=1}^{\lambda} C_{\Phi\left(p_{i}^{\ell_{i}}\right)}$, where the $p_{i}$ are distinct odd primes and $\ell_{i} \geq 1$.

Step 2: Either (i) reduce the order of some $C_{\Phi\left(p_{i}^{\ell_{i}}\right)}$ to $\frac{1}{2} \Phi\left(p_{i}^{\ell_{i}}\right)$, where $p_{i}$ is chosen so that the 2 -valuation of $p_{i}-1$ is minimal; or (ii) take the direct product with $C_{2^{\ell}}, \ell \geq 0$.

Notice that we may simply stop at Step 1.

Our second observation relates to Step 2 in Theorem 3.3. Obviously Step 2 involves factoring out of the group taken in Step 1 an elementary abelian 2-subgroup. An easy extension of Lemma 3.1 establishes

Theorem 3.5. If $H$ is any elementary abelian 2-subgroup of the group described in Step 1 of Theorem 3.3, then the quotient of this group by $H$ may be achieved by a suitably chosen Step 2.

\section{Examples and supplementary results}

We first give some examples of realizability and non-realizability.

Example 4.1. We may realize the group $G=\mathbb{Z} / 4 \oplus \mathbb{Z} / 2 \oplus \mathbb{Z} / 3$ as $\mathcal{G}(N)$ for $N \in \mathcal{N}_{1}$. For, in multiplicative notation, $G=C_{4} \times C_{\Phi\left(3^{2}\right)}$ so $G \cong(\mathbb{Z} / t)^{*} /\{ \pm 1\}$ for $t=144$. Of course, other values of $t$ will also serve, e.g. $t=104,112$. It is shown in $[\mathbf{1}]$ or [4] how any $t$ may be realized by a group $N$ in $\mathcal{N}_{1}$.

Example 4.2. We cannot realize the group $\mathbb{Z} / 5 \oplus \mathbb{Z} / 9$ as $\mathcal{G}(N)$ for $N \in \mathcal{N}_{1}$. This follows from the fact that 90 is not a value taken by the Euler totient function $\Phi$. For if $\Phi(t)=90$, then we easily eliminate $t=p, p^{2}, p^{3}$ ( $p$ odd); but if $t=2^{\ell+2} p^{m}(\ell \geq 0)$ or $t=m p q$ ( $q$ odd $)$, then $4 \mid \Phi(t)$.

On the other hand, we can realize $\mathbb{Z} / 5 \oplus \mathbb{Z} / 9$ as $\mathcal{G}(N)$ for $N \in \mathcal{N}_{2}$. For if we start with $C_{\Phi(11)} \times C_{\Phi(19)}=C_{10} \times C_{18}$, we reduce the order of both factors to get $C_{5} \times C_{9}$ and Step 3 is void. This realization amounts to choosing $N$ so that $t=836$ and $P=\{11,19\}$. (We will see later how to realize any $(t, P)$ by a group $N$ in $\mathcal{N}_{2}$ ).

We next prove a theorem on the realizability of cyclic groups of prime power order. 
Theorem 4.3. Let $p$ be a prime number and $m \geq 1$. Then $C_{p^{m}}$ may be realized as $\mathcal{G}(N), N \in \mathcal{N}_{2}$, if and only if $p=2, \bar{p}=3$ or $2 p^{m}+1$ is prime.

Proof: It is plain that if $p=2, p=3$, or $2 p^{m}+1$ is prime, then $C_{p^{m}}$ may even be realized as $\mathcal{G}(N)$ for some $N$ in $\mathcal{N}_{1}$. To prove the converse, suppose that $C_{p^{m}}$ is obtained from $(\mathbb{Z} / t)^{*}$ by factoring out some elementary abelian 2-subgroup $H$. We assume henceforth that $p \neq 2$. Let $t=2^{\ell} \prod_{i=1}^{\lambda} p_{i}^{\ell_{i}}$, where each $p_{i}$ is odd and $\ell_{i} \geq 1$. Since $|\mathcal{G}(N)|$ is to be odd, it is clear that $\ell=0,1$ or 2 (the case $\ell=1$ can be ignored in practice) and that all possible reductions of order must take place. Thus

$$
C_{p^{m}} \cong \prod_{i=1}^{\lambda} C_{\frac{1}{2}\left(p_{i}-1\right) p_{i}^{\ell_{i}-1}}
$$

If any $\ell_{i} \geq 2$ then (4.1) implies that $p_{i}=p$ and $\frac{1}{2}\left(p_{i}-1\right)=1$, so $p=p_{i}=3$. If each $\ell_{i}=1$, then each group on the right of (4.1) is a $p$-group, so there can be only one non-trivial factor, say the $i$ th factor, yielding $\frac{1}{2}\left(p_{i}-1\right)=p^{m}$. Thus $2 p^{m}+1=p_{i}$ is prime.

\section{Remarks.}

(a) Notice that, in fact, $t$ can only have, at most, two odd prime factors, namely 3 and $2 p^{m}+1$.

(b) We find a source of genera which are cyclic 2 -groups by taking $t$ to be a Fermat prime. Of course, we may take $t$ to be a product of distinct Fermat primes to yield genera which are non-cyclic 2-groups.

(c) Mendelsohn (see [6]) has proved that there exist infinitely many primes $p$ such that $2^{n} p$ is not a value of the $\Phi$-function, for any $n \geq 1$. For such primes $p$, no group of order $2^{m} p, m>0$, can be realizable.

We close by showing how to realize a pair $(t, P)$, where $P \subseteq T_{t}$, by a group $N$ in $\mathcal{N}_{2}$. We first take $P=\varnothing$ and realize $t$ by a group $N$ in $\mathcal{N}_{1}$. The procedure given in $[\mathbf{1}]$ or $[4]$ is as follows. Let $t$ be odd, say, $t=p_{1}^{\ell_{1}} \ldots p_{\lambda}^{\ell_{\lambda}}$. Set $T N=\mathbb{Z} / n$, where $n=p_{1}^{\ell_{1}+1} \ldots p_{\lambda}^{\ell_{\lambda}+1}$ and let $F N=\langle\xi\rangle$ act on $T N$ by $\xi \cdot a=u a$, where $u=1+p_{1} \ldots p_{\lambda}$. If $N$ is the semidirect product for this action, then $N$ is nilpotent and $\mathcal{G}(N)=(\mathbb{Z} / t)^{*} /\{ \pm 1\}$. Now let $t$ be even, say $t=2^{\ell} p_{1}^{\ell_{1}} \ldots p_{\lambda}^{\ell_{\lambda}}$. Set $T N=\mathbb{Z} / n$, where $n=2^{\ell+2} p_{1}^{\ell_{1}+1} \ldots p_{\lambda}^{\ell_{\lambda}+1}$ and let $F N=\langle\xi\rangle$ act on $T N$ 
by $\xi \cdot a=u a$, where $u=1+4 p_{1} \ldots p_{\lambda}$. If $N$ is the semidirect product for this action, then $N$ is nilpotent and $\mathcal{G}(N)=(\mathbb{Z} / t)^{*} /\{ \pm 1\}$. Certainly, in both cases, $N \in \mathcal{N}_{1}$.

We now pass to the general case; as indicated earlier, we will be able to realize $(t, P)$ by a group in $\mathcal{N}_{2}$ of the form $N_{1} \times N_{2}$, with $N_{1}, N_{2}$ in $\mathcal{N}_{1}$. We first realize $t$ just as above by a group $N_{1}$ in $\mathcal{N}_{1}$. The group $N_{2}$ is constructed just as $N_{1}$ except that, for the order $n^{\prime}$ of $T N_{2}=\mathbb{Z} / n^{\prime}$, we raise the power of those primes outside $P$ (including, perhaps, the prime 2) by 1 . Of course, if $P=T_{t}$, then this recipe yields $N_{2}=N_{1}$.

Example 4.4. Let $t=165, P=\varnothing$. Then we construct $N$ in $\mathcal{N}_{1}$ by taking $T N=\mathbb{Z} / n, n=27225$; and $F N=\langle\xi\rangle$ acts on $T N$ by $\xi \cdot a=166 a$. We may describe $N$ as

$$
N=\left\langle x, y \mid x^{27225}=1, y x y^{-1}=x^{166}\right\rangle .
$$

Then $\mathcal{G}(N)=(\mathbb{Z} / 165)^{*} /\{ \pm 1\}=C_{4} \times C_{10}$.

Now take $P=\{5\}$. Then we construct $N_{1}$ as $N$ was constructed above. However, for $N_{2}$, we replace 27225 by $27225 \cdot 33=898425$. Then

$$
\mathcal{G}\left(N_{1} \times N_{2}\right)=(\mathbb{Z} / 165)^{*} /\langle-1,34\rangle=C_{2} \times C_{10} .
$$

\section{References}

1. C. Casacuberta and P. Hilton, Calculating the Mislin genus for a certain family of nilpotent groups, Comm. Algebra 19(7) (1991), 2051-2069.

2. P. Hilton And G. Mislin, On the genus of a nilpotent group with finite commutator subgroup, Math. Z. 146 (1976), 201-211.

3. P. Hilton And D. Scevenels, Calculating the genus of a direct product of certain nilpotent groups, Publ. Mat. 39 (1995), 241-261.

4. P. Hilton ANd C. Schuck, On the structure of nilpotent groups of a certain type, Topol. Methods Nonlinear Anal. 1 (1993), 323-327.

5. G. Mislin, Nilpotent groups with finite commutator subgroups, in "Localization in Group Theory and Homotopy Theory," Lecture Notes in Math. 418, Springer-Verlag, 1974, pp. 103-120. 
6. P. Ribenboim, "The New Book of Prime Number Records," Springer-Verlag, 1996.

Peter Hilton:

Department of Mathematical Sciences

State University of New York

Binghamton, NY 13902-6000

U.S.A.
Dirk Scevenels:

Departement Wiskunde

Katholieke Universiteit Leuven

Celestijnenlaan 200 B

B-3001 Heverlee

BELGIUM

Primera versió rebuda el 20 de Maig de 1996, darrera versió rebuda el 17 d'Abril de 1997 